\title{
TECHNICAL LEMMAS
}

\section{By YUAN WU AND Ying ZHANG}

University of California San Diego and University of Iowa

Lemma 0.1. Suppose $\tau=\tau_{0}$ or $\tau \in \Omega_{n}^{\prime}$, then under $C 2$ and $C 6$ in the main paper, the following two properties hold for $F(s, t), F_{1}(s)$ and $F_{2}(t)$ with $\tau=\left(F, F_{1}, F_{2}\right)$.

(1) $F(s, t)$ is nondecreasing in both $s$ and $t . F_{1}(s)-F(s, t)$ is nondecreasing in $s$ and nonincreasing in $t . F_{2}(t)-F(s, t)$ is nondecreasing in $t$ and nonincreasing in $s .1-F_{1}(s)-F_{2}(t)+F(s, t)$ is nonincreasing in both $s$ and $t$.

(2) $F(s, t), F_{1}(s)-F(s, t), F_{2}(t)-F(s, t)$ and $1-F_{1}(s)-F_{2}(t)+F(s, t)$ all have positive lower bounds.

Proof. First, we verify the two properties for $\tau=\tau_{0}$.

Property (1) is obviously true by the properties of any joint distribution. Under C2 in the main paper and by $(s, t) \in\left[l_{1}, u_{1}\right] \times\left[l_{2}, u_{2}\right]$,

$$
\begin{aligned}
& F(s, t)=F_{0}(s, t)=P\left(T_{1} \leq s, T_{2} \leq t\right) \geq P\left(L_{1}<T_{1} \leq s, L_{2}<T_{2} \leq t\right) \\
& \geq\left(s-L_{1}\right)\left(t-L_{2}\right) \min _{s, t} \frac{\partial^{2} F_{0}(s, t)}{\partial s \partial t} \geq\left(l_{1}-L_{1}\right)\left(l_{2}-L_{2}\right) b_{0}, \\
& F_{1}(s)-F(s, t)=F_{0,1}(s)-F_{0}(s, t)=P\left(T_{1} \leq s, T_{2}>t\right) \\
& \geq P\left(L_{1}<T_{1} \leq s, t<T_{2} \leq U_{2}\right) \\
& \geq\left(s-L_{1}\right)\left(U_{2}-t\right) \min _{s, t} \frac{\partial^{2} F_{0}(s, t)}{\partial s \partial t} \geq\left(l_{1}-L_{1}\right)\left(U_{2}-u_{2}\right) b_{0}, \\
& F_{2}(t)-F(s, t)=F_{0,2}(t)-F_{0}(s, t)=P\left(T_{1}>s, T_{2} \leq t\right) \\
& \geq P\left(s<T_{1} \leq U_{1}, L_{2}<T_{2} \leq t\right) \\
& \geq\left(U_{1}-s\right)\left(t-L_{2}\right) \min _{s, t} \frac{\partial^{2} F_{0}(s, t)}{\partial s \partial t} \geq\left(U_{1}-u_{1}\right)\left(l_{2}-L_{2}\right) b_{0},
\end{aligned}
$$


and

$$
\begin{aligned}
1-F_{1}(s)-F_{2}(t)+F(s, t) & =1-F_{0,1}(s)-F_{0,2}(t)+F_{0}(s, t) \\
& =P\left(T_{1}>s, T_{2}>t\right) \\
& \geq P\left(s<T_{1} \leq U_{1}, t<T_{2} \leq U_{2}\right) \\
& \geq\left(U_{1}-s\right)\left(U_{2}-t\right) \min _{s, t} \frac{\partial^{2} F_{0}(s, t)}{\partial s \partial t} \\
& \geq\left(U_{1}-u_{1}\right)\left(U_{2}-u_{2}\right) b_{0} .
\end{aligned}
$$

Second, we verify the two properties for $\tau \in \Omega_{n}^{\prime}$.

Remark 2.1 in the main paper indicates that $\Omega_{n}^{\prime} \subset \mathcal{F}$ in $\left[l_{1}, u_{1}\right] \times\left[l_{2}, u_{2}\right]$, hence any $\tau=\left(F, F_{1}, F_{2}\right) \in \Omega_{n}^{\prime}$ satisfies property (1).

Given C6 and the 4th inequality in (3.1) of the main paper, we can have

$$
\begin{aligned}
\frac{\partial^{2} F(s, t)}{\partial s \partial t} & \geq \min _{i, j} \frac{\alpha_{i+1, j+1}-\alpha_{i, j+1}-\alpha_{i+1, j}+\alpha_{i, j}}{\max _{i_{1}} \Delta_{i_{1}}^{(u)} \max _{j_{1}} \Delta_{j_{1}}^{(v)}} \\
& \geq \min _{i, j} \frac{\alpha_{i+1, j+1}-\alpha_{i, j+1}-\alpha_{i+1, j}+\alpha_{i, j}}{\frac{\min _{i_{1}} \Delta_{i_{1}}^{(u)}}{l} \frac{\min _{j_{1}} \Delta_{j_{1}}^{(v)}}{l}} \frac{\frac{\min _{i_{1}} \Delta_{i_{1}}^{(u)}}{l} \frac{\min _{j_{1}} \Delta_{j_{1}}^{(v)}}{l}}{\max _{i_{1}} \Delta_{i_{1}}^{(u)} \max _{j_{1}} \Delta_{j_{1}}^{(v)}} \\
& \geq K .
\end{aligned}
$$

(0.1) implies that for $(s, t) \in\left[l_{1}, u_{1}\right] \times\left[l_{2}, u_{2}\right]$

$$
\begin{aligned}
F(s, t) & \geq F(s, t)-F\left(s, L_{2}\right)-F\left(L_{1}, t\right)+F\left(L_{1}, L_{2}\right) \\
& =\int_{s}^{L_{1}} \int_{t}^{L_{2}} \frac{\partial^{2} F(x, y)}{\partial x \partial y} d y d x \\
& \geq\left(s-L_{1}\right)\left(t-L_{2}\right) \min _{s, t} \frac{\partial^{2} F(s, t)}{\partial s \partial t} \geq\left(l_{1}-L_{1}\right)\left(l_{2}-L_{2}\right) K .
\end{aligned}
$$

(0.1) also implies that

$$
\begin{aligned}
F_{1}(s)-F(s, t) & \geq F\left(s, U_{2}\right)-F(s, t) \\
& \geq F\left(s, U_{2}\right)-F(s, t)-F\left(L_{1}, U_{2}\right)+F\left(L_{1}, t\right) \\
& \geq\left(l_{1}-L_{1}\right)\left(U_{2}-u_{2}\right) K
\end{aligned}
$$

In addition, (0.1) implies that

$$
F_{2}(t)-F(s, t) \geq\left(U_{1}-u_{1}\right)\left(l_{2}-L_{2}\right) K .
$$


Finally, (0.1) results in

$$
\begin{aligned}
1-F_{1}(s)-F_{2}(t)+F(s, t) & \geq F\left(U_{1}, U_{2}\right)-F\left(s, U_{2}\right)-F\left(U_{1}, t\right)+F(s, t) \\
& \geq\left(U_{1}-u_{1}\right)\left(U_{2}-u_{1}\right) K .
\end{aligned}
$$

Lemma 0.2. Suppose $g(x, y)$ is a bivariate function in the closed region $\left[L_{1}, U_{1}\right] \times\left[L_{2}, U_{2}\right]$ with continuous derivatives up to order $w, \nabla_{m}^{w} g=$ $\frac{\partial^{w} g(x, y)}{\partial x^{m} y^{w-m}}$ for $m=1,2, \ldots, w$. Then there exists a bivariate function made by a linear combination of tensor B-spline basis functions,

$A g(x, y)=\sum_{i=1}^{p} \sum_{j=1}^{q} \alpha_{i, j} N_{i}^{(1), l}(x) N_{j}^{(2), l}(y)$, with order $l \geq w+1$ for every B-spline basis function and $\left\{N_{i}^{(1), l}\right\}_{i=1}^{p}$ having knot sequence $\left\{u_{i}\right\}_{i=1}^{p+l}$ satisfying $L_{1}=u_{1}=\cdots=u_{l}<u_{l+1}<\cdots<u_{p}<u_{p+1}=\cdots=u_{p+l}=U_{1}$, $\left\{N_{j}^{(2), l}\right\}_{j=1}^{q}$ having knot sequence $\left\{v_{j}\right\}_{j=1}^{q+l}$ satisfying $L_{2}=v_{1}=\cdots=v_{l}<$ $v_{l+1}<\cdots<v_{q}<v_{q+1}=\cdots=v_{q+l}=U_{2}$, such that for some constant $K>0$

$$
\|g-A g\|_{\infty} \leq K|T|^{w}\left(\|g\|_{w, \infty}\right),
$$

where $|T|=\max \left\{\max _{l \leq i \leq p}\left(u_{i+1}-u_{i}\right), \max _{l \leq j \leq q}\left(v_{j+1}-v_{j}\right)\right\}$, and $\|g\|_{w, \infty}=\max _{0 \leq m \leq w}\left\|\frac{\partial^{w} g}{\partial x^{m} \partial y^{w-m}}\right\|_{\infty}$.

PROOF. The proof of this lemma closely follows the arguments for justifying Jackson Theorem in De Boor $(2001, \mathrm{p} 149)$. We define $\omega(g ; h)=$ $\max \left\{\left|g\left(x_{1}, y_{1}\right)-g\left(x_{2}, y_{2}\right)\right|:\left|x_{1}-x_{2}\right| \leq h,\left|y_{1}-y_{2}\right| \leq h, x_{1}, x_{2} \in\left[L_{1}, U_{1}\right], y_{1}, y_{2}\right.$ $\left.\in\left[L_{2}, U_{2}\right]\right\}$. Then $\omega(g ; h)$ is a monotone and subadditivity function of $h$, that is, $\omega\left(g ; h_{1}\right) \leq \omega\left(g ; h_{1}+h_{2}\right) \leq \omega\left(g ; h_{1}\right)+\omega\left(g ; h_{2}\right)$ for nonnegative $h_{1}$ and $h_{2}$. The monotonicity of $\omega(g ; h)$ is automatically true by the definition. The proof of subadditivity is as follows.

For any $\left(x_{1}, y_{1}\right)$ and $\left(x_{2}, y_{2}\right)$ with $\left|x_{1}-x_{2}\right| \leq h_{1}+h_{2}$ and $\left|y_{1}-y_{2}\right| \leq$ $h_{1}+h_{2}$, we can find $\left(x_{3}, y_{3}\right)$ such that $\left|x_{1}-x_{3}\right| \leq h_{1},\left|y_{1}-y_{3}\right| \leq h_{1}$ and $\left|x_{2}-x_{3}\right| \leq h_{2},\left|y_{2}-y_{3}\right| \leq h_{2}$. Therefore, for any $\left|x_{1}-x_{2}\right| \leq h_{1}+h_{2}$ and $\left|y_{1}-y_{2}\right| \leq h_{1}+h_{2}$, we have

$$
\begin{aligned}
\left|g\left(x_{1}, y_{1}\right)-g\left(x_{2}, y_{2}\right)\right| \leq & \left|g\left(x_{1}, y_{1}\right)-g\left(x_{3}, y_{3}\right)\right|+\left|g\left(x_{3}, y_{3}\right)-g\left(x_{2}, y_{2}\right)\right| \\
\leq & \max _{\substack{\left|x_{1}-x_{3}\right| \leq h_{1} \\
\left|y_{1}-y_{3}\right| \leq h_{1}}}\left|g\left(x_{1}, y_{1}\right)-g\left(x_{3}, y_{3}\right)\right| \\
& +\max _{\substack{\left|x_{2}-x_{3}\right| \leq h_{2} \\
\left|y_{2}-y_{3}\right| \leq h_{2}}}\left|g\left(x_{3}, y_{3}\right)-g\left(x_{2}, y_{2}\right)\right| \\
= & \omega\left(g ; h_{1}\right)+\omega\left(g ; h_{2}\right) .
\end{aligned}
$$


By $(0.2), \omega\left(g ; h_{1}+h_{2}\right) \leq \omega\left(g ; h_{1}\right)+\omega\left(g ; h_{2}\right)$ for nonnegative $h_{1}$ and $h_{2}$, that is, the subadditivity of $\omega(g ; h)$ holds.

By choosing $\tau_{1}<\tau_{2}<\cdots<\tau_{p}$ in $\left[L_{1}, U_{1}\right]$ and $\xi_{1}<\xi_{2}<\cdots<\xi_{q}$ in $\left[L_{2}, U_{2}\right]$, we can construct a linear combination of the tensor $B$-spline basis functions $\mathrm{Ag}$ to approximate the smooth function $g$ on $\left[L_{1}, U_{1}\right] \times\left[L_{2}, U_{2}\right]$ as follows.

$$
A g(x, y)=\sum_{i=1}^{p} \sum_{j=1}^{q} g\left(\tau_{i}, \xi_{j}\right) N_{i}^{(1), l}(x) N_{j}^{(2), l}(y)
$$

For $(\hat{x}, \hat{y})$ in $\left[u_{j_{1}}, u_{j_{1}+1}\right] \times\left[v_{j_{2}}, v_{j_{2}+1}\right] \in\left[L_{1}, U_{1}\right] \times\left[L_{2}, U_{2}\right]$,

$$
A g(\hat{x}, \hat{y})=\sum_{i=j_{1}+1-l}^{j_{1}} \sum_{j=j_{2}+1-l}^{j_{2}} g\left(\tau_{i}, \xi_{j}\right) N_{i}^{(1), l}(\hat{x}) N_{j}^{(2), l}(\hat{y}),
$$

due to the fact that the supports of the $B$-spline basis functions only cover a part of the knot intervals. Since the $B$-spline basis functions sum to one, we have

$$
\begin{aligned}
g(\hat{x}, \hat{y}) & =g(\hat{x}, \hat{y}) \sum_{i=j_{1}+1-l}^{j_{1}} N_{i}^{(1), l}(\hat{x}) \\
& =g(\hat{x}, \hat{y}) \sum_{i=j_{1}+1-l}^{j_{1}}\left\{\sum_{j=j_{2}+1-l}^{j_{2}} N_{j}^{(2), l}(\hat{y})\right\} N_{i}^{(1), l}(\hat{x}) \\
& =g(\hat{x}, \hat{y}) \sum_{i=j_{1}+1-l}^{j_{1}} \sum_{j=j_{2}+1-l}^{j_{2}} N_{i}^{(1), l}(\hat{x}) N_{j}^{(2), l}(\hat{y}) .
\end{aligned}
$$

Subtracting (0.3) from (0.4) yields,

$$
g(\hat{x}, \hat{y})-A g(\hat{x}, \hat{y})=\sum_{i=j_{1}+1-l}^{j_{1}} \sum_{j=j_{2}+1-l}^{j_{2}}\left\{g(\hat{x}, \hat{y})-g\left(\tau_{i}, \xi_{j}\right)\right\} N_{i}^{(1), l}(\hat{x}) N_{j}^{(2), l}(\hat{y}) .
$$

Hence,

$$
\begin{aligned}
|g(\hat{x}, \hat{y})-A g(\hat{x}, \hat{y})| & \leq \sum_{i=j_{1}+1-l-l j=j_{2}+1-l}^{j_{1}} \sum^{j_{2}}\left|g(\hat{x}, \hat{y})-g\left(\tau_{i}, \xi_{j}\right)\right| N_{i}^{(1), l}(\hat{x}) N_{j}^{(2), l}(\hat{y}) \\
& \leq \max _{\substack{j_{1}+1-l \leq i \leq j_{1} \\
j_{2}+1-l \leq j \leq j_{2}}}\left|g(\hat{x}, \hat{y})-g\left(\tau_{i}, \xi_{j}\right)\right| .
\end{aligned}
$$


We specifically choose the sequences of $\left\{\tau_{i}\right\}_{i=1}^{p}$ and $\left\{\xi_{j}\right\}_{j=1}^{q}$ as follows

$$
\begin{aligned}
& \tau_{i}=\left\{\begin{array}{l}
u_{1}+\frac{(i-1)\left(u_{l+1}-u_{l}\right)}{l}, i=1, \ldots, l, \\
u_{i}, i=l+1, \ldots, p .
\end{array}\right. \\
& \xi_{j}=\left\{\begin{array}{l}
v_{1}+\frac{(j-1)\left(v_{l+1}-v_{l}\right)}{l}, j=1, \ldots, l, \\
v_{j}, j=l+1, \ldots, q,
\end{array}\right.
\end{aligned}
$$

Then (0.5) and (0.6) imply $\left|\tau_{i}-u_{i}\right| \leq|T|$ and $\left|\xi_{j}-v_{j}\right| \leq|T|$ for $i=$ $1, \ldots, p$ and $j=1, \ldots, q$. We also know $\left|u_{i}-\hat{x}\right| \leq u_{j_{1}+1}-u_{j_{1}-l+1} \leq l|T|$ for $j_{1}-l<i \leq j_{1}$ and $\hat{x} \in\left[u_{j_{1}}, u_{j_{1}+1}\right]$ and $\left|v_{j}-\hat{y}\right| \leq v_{j_{2}+1}-v_{j_{2}-l+1} \leq l|T|$ for $j_{2}-l<j \leq j_{2}$ and $\hat{y} \in\left[v_{j_{2}}, v_{j_{2}+1}\right]$.

Then for $j_{1}-l<i \leq j_{1}$ and $\hat{x} \in\left[u_{j_{1}}, u_{j_{1}+1}\right]$

$$
\left|\tau_{i}-\hat{x}\right| \leq(l+1)|T|,
$$

and for $j_{2}-l<j \leq j_{2}$ and $\hat{y} \in\left[v_{j_{2}}, v_{j_{2}+1}\right]$

$$
\left|\xi_{j}-\hat{y}\right| \leq(l+1)|T|
$$

Hence,

$$
\begin{aligned}
& \max _{\substack{j_{1}+1-l \leq i \leq j_{1} \\
j_{2}+1-l \leq j \leq j_{2}}}\left|g(\hat{x}, \hat{y})-g\left(\tau_{i}, \xi_{j}\right)\right| \leq \max \left\{\left|g\left(x_{1}, y_{1}\right)-g\left(x_{2}, y_{2}\right)\right|:\right. \\
& \\
& \left.\left|x_{1}-x_{2}\right| \leq(l+1)|T|,\left|y_{1}-y_{2}\right| \leq(l+1)|T|\right\} \\
& =\omega(g ;(l+1)|T|)=(l+1) \omega(g ;|T|),
\end{aligned}
$$

where the last inequality is due to the subadditivity property of $\omega(g ; h)$.

(0.7) implies that

$$
\|g-A g\|_{\infty}=\sup _{\substack{L_{1} \leq x \leq U_{1} \\ L_{2} \leq y \leq U_{2}}}|g(x, y)-A g(x, y)| \leq(l+1) \omega(g ;|T|),
$$

which means the distance between $g$ and $\psi_{l, l}$

$$
d\left(g, \psi_{l, l}\right)=\inf _{f \in \psi_{l, l}}\|g-f\|_{\infty} \leq(l+1) \omega(g ;|T|),
$$

where $\psi_{l, l}$ denotes the space of all linear combinations of the tensor $B$-spline basis functions with order $l$ for every basis function. Because the distance of 
function $g$ from $\psi_{l, l}$ is the same as the distance of the function $g-f$ from $\psi_{l, l}$ for $f \in \psi_{l, l},(0.8)$ implies

$$
d\left(g, \psi_{l, l}\right)=d\left(g-f, \psi_{l, l}\right) \leq(l+1) \omega(g-f,|T|) .
$$

Furthermore, since $g$ has bounded partial derivatives, then

$$
\begin{aligned}
\omega(g-f,|T|)= & \max _{\left|x_{1}-x_{2}\right| \leq|T|}\left|(g-f)\left(x_{1}, y_{1}\right)-(g-f)\left(x_{2}, y_{2}\right)\right| \\
\leq & \max _{\left|y_{1}-y_{2}\right| \leq|T|}\left|(g-f)\left(x_{1}, y_{1}\right)-(g-f)\left(x_{1}, y_{2}\right)\right| \\
& +\max _{\left|x_{1}-x_{2}\right| \leq|T|}\left|(g-f)\left(x_{1}, y_{2}\right)-(g-f)\left(x_{1}, y_{2}\right)\right| \\
\leq & \left\|\frac{\partial(g-f)}{\partial y}\right\|_{\infty}|T|+\left\|\frac{\partial(g-f)}{\partial x}\right\|_{\infty}|T| .
\end{aligned}
$$

Therefore, by (0.9)

$$
d\left(g, \psi_{l, l}\right) \leq(l+1)|T|\left(\left\|\frac{\partial(g-f)}{\partial y}\right\|_{\infty}+\left\|\frac{\partial(g-f)}{\partial x}\right\|_{\infty}\right) .
$$

Since $\psi_{l, l-1}=\left\{\frac{\partial f}{\partial y}: f \in \psi_{l, l}\right\}$ and $\psi_{l-1, l}=\left\{\frac{\partial f}{\partial x}: f \in \psi_{l, l}\right\},(0.10)$ implies

$$
d\left(g, \psi_{l, l}\right) \leq(l+1)|T|\left\{d\left(\frac{\partial g}{\partial x}, \psi_{l-1, l}\right)+d\left(\frac{\partial g}{\partial y}, \psi_{l, l-1}\right)\right\} .
$$

Iterating the same derivation for (0.11) leads to

$$
\begin{aligned}
& d\left(g, \psi_{l, l}\right) \\
& \leq K|T|^{w-1}\left\{d\left(\frac{\partial^{w-1} g}{\partial x^{w-1}}, \psi_{l-w+1, l}\right)\right. \\
&\left.+d\left(\frac{\partial^{w-1} g}{\partial x^{w-2} \partial y}, \psi_{l-w+2, l-1}\right)+\cdots+d\left(\frac{\partial^{w-1} g}{\partial y^{w-1}}, \psi_{l, l-w+1}\right)\right\} \\
& \leq K|T|^{w-1}\left\{\omega\left(\frac{\partial^{w-1} g}{\partial x^{w-1}},|T|\right)+\omega\left(\frac{\partial^{w-1} g}{\partial x^{w-2} \partial y},|T|\right)\right. \\
&\left.\quad+\cdots+\omega\left(\frac{\partial^{w-1} g}{\partial y^{w-1}},|T|\right)\right\} \\
& \leq K|T|^{w}\left\{\left\|\frac{\partial^{w} g}{\partial x^{w}}\right\|_{\infty}+\left\|\frac{\partial^{w} g}{\partial x^{w-1} \partial y}\right\|_{\infty}+\cdots+\left\|\frac{\partial^{w} g}{\partial y^{w}}\right\|_{\infty}\right\} \\
& \leq K|T|^{w} \max _{0 \leq m \leq w}\left\|\frac{\partial^{w} g}{\partial x^{m} \partial y^{w-m}}\right\|_{\infty} \cdot
\end{aligned}
$$


Lemma 0.3. Let $p_{n}=O\left(n^{v}\right)$ and $q_{n}=O\left(n^{v}\right)$. If C2, C3 and C6 in the main paper hold, there exists $\tau_{n}=\left(F_{n}, F_{n, 1}, F_{n, 2}\right) \in \Omega_{n}^{\prime}$, such that $\| F_{n}-$ $F_{0}\left\|_{\infty} \leq K\left(n^{-p v}\right),\right\| F_{n, 1}-F_{0,1} \|_{\infty} \leq K\left(n^{-p v}\right)$ and $\left\|F_{n, 2}-F_{0,2}\right\|_{\infty} \leq K\left(n^{-p v}\right)$.

Proof. Suppose the spline coefficients of $F_{n}, F_{n, 1}$ and $F_{n, 2}$ are chosen as $\alpha_{i, j}=F_{0}\left(\tau_{i}, \xi_{j}\right), \beta_{i}=F_{0,1}\left(\tau_{i}\right)$ and $\gamma_{j}=F_{0,2}\left(\xi_{j}\right)$, where $\tau_{i}, i=1, \ldots, p_{n}$ and $\xi_{j}, j=1, \ldots, q_{n}$ are defined by $(0.5)$ and (0.6) in the proof of Lemma 0.2. Given C3, C6 in the main paper, Jackson Theorem in De boor (2001, p149) and Lemma 0.2 , it is easily seen that that $\left\|F_{n}-F_{0}\right\|_{\infty} \leq K\left(n^{-p v}\right)$, $\left\|F_{n, 1}-F_{0,1}\right\|_{\infty} \leq K\left(n^{-p v}\right)$, and $\left\|F_{n, 2}-F_{0,2}\right\|_{\infty} \leq K\left(n^{-p v}\right)$.

To complete the proof, it remains to show that $\alpha_{i, j}, \beta_{i}$ and $\gamma_{j}$ satisfy the inequalities in (3.1) in the main paper.

(i) $\alpha_{1,1}=F_{0}\left(\tau_{1}, \xi_{1}\right) \geq 0$.

(ii) $\alpha_{1, j+1}-\alpha_{1, j}=F_{0}\left(\tau_{1}, \xi_{j+1}\right)-F_{0}\left(\tau_{1}, \xi_{j}\right) \geq 0$.

(iii) $\alpha_{i+1,1}-\alpha_{i, 1}=F_{0}\left(\tau_{i+1}, \xi_{1}\right)-F_{0}\left(\tau_{i}, \xi_{1}\right) \geq 0$.

(iv) $\frac{\left(\alpha_{i+1, j+1}-\alpha_{i+1, j}\right)-\left(\alpha_{i, j+1}-\alpha_{i, j}\right)}{\frac{\min _{i_{1}} \Delta_{i_{1}}^{(u)} \min _{j_{1}} \Delta_{j_{1}}^{(v)}}{{ }^{l}}} \geq \frac{\alpha_{i+1, j+1}-\alpha_{i, j+1}-\alpha_{i+1, j}+\alpha_{i, j}}{\left(\tau_{i+1}-\tau_{i}\right)\left(\xi_{j+1}-\xi_{i}\right)}$ $=\frac{F_{0}\left(\tau_{i+1}, \xi_{j+1}\right)-F_{0}\left(\tau_{i}, \xi_{j+1}\right)-F_{0}\left(\tau_{i+1}, \xi_{j}\right)+F_{0}\left(\tau_{i}, \xi_{j}\right)}{\left(\tau i+1-\tau_{i}\right)\left(\xi_{j+1}-\xi_{i}\right)} \geq \min _{\substack{s \in\left[L_{1}, U_{1}\right] \\ t \in\left[L_{2}, U_{2}\right]}} \frac{\partial^{2} F_{0}(s, t)}{\partial s \partial t}=$ $b_{0}$, by $\mathrm{C} 2$ in the main paper.

(v) $\beta_{1}-\alpha_{1, q_{n}}=F_{0,1}\left(\tau_{1}\right)-F_{0}\left(\tau_{1}, \xi_{q_{n}}\right) \geq 0$.

(vi) $\beta_{i+1}-\beta_{i}-\left(\alpha_{i+1, q_{n}}-\alpha_{i, q_{n}}\right)=F_{0,1}\left(\tau_{i+1}\right)-F_{0,1}\left(\tau_{i}\right)-\left(F_{0}\left(\tau_{i+1}, \xi_{q_{n}}\right)-\right.$ $\left.F_{0}\left(\tau_{i}, \xi_{q_{n}}\right)\right) \geq 0$.

(vii) $\gamma_{1}-\alpha_{p_{n}, 1}=F_{0,2}\left(\xi_{1}\right)-F_{0}\left(\tau_{p_{n}}, \xi_{1}\right) \geq 0$.

(viii) $\gamma_{j+1}-\gamma_{j}-\left(\alpha_{p_{n}, j+1}-\alpha_{p_{n}, j}\right)=F_{0,2}\left(\xi_{j+1}\right)-F_{0,1}\left(\xi_{j}\right)-\left(F_{0}\left(\tau_{p_{n}}, \xi_{j+1}\right)-\right.$ $\left.F_{0}\left(\tau_{p_{n}}, \xi_{j}\right)\right) \geq 0$

(ix) $1-\beta_{p_{n}}-\gamma_{q_{n}}+\alpha_{p_{n}, q_{n}}=1-F_{0,1}\left(\tau_{p_{n}}\right)-F_{0,2}\left(\xi_{q_{n}}\right)+F_{0}\left(\tau_{p_{n}}, \xi_{q_{n}}\right) \geq 0$.

Lemma 0.4. Let $S$ be a sphere in $R^{n}$ with radius $\left(n^{1 / 2} \sigma\right)$, that is, $S=$ $\left\{x=\left(x_{1}, \ldots, x_{n}\right) \in R^{n}: \sum_{i=1}^{n} x_{i}^{2} \leq n \sigma^{2}\right\}$. Let $\|\cdot\|_{\infty}$ be the usual $L_{\infty}$-norm in $R^{n}$. Then $\log N\left(\epsilon, S,\|\cdot\|_{\infty}\right) \leq K n \log (\sigma / \epsilon)$, for some constant $K>0$ and $\epsilon<\sigma$.

Proof. The proof follows along the same lines as for the proof of Lemma 5 in Shen and Wong (1994).

Lemma 0.5. $\Theta_{\delta}=\left\{\phi: \phi(s, t)=\sum_{i=1}^{p} \sum_{j=1}^{q} \alpha_{i, j} N_{i}^{(1), l}(s) N_{j}^{(2), l}(t),\|\phi\|_{\infty}\right.$ $<\delta\},\left\{N_{i}^{(1), l}\right\}_{i=1}^{p}$ and $\left\{N_{j}^{(2), l}\right\}_{j=1}^{q}$ are two sets of B-spline basis functions 
with the knot sequence $\left\{u_{i}\right\}_{i=1}^{p+l}$ satisfying $L_{1}=u_{1}=\cdots=u_{l}<u_{l+1}<$ $\cdots<u_{p}<u_{p+1}=\cdots=u_{p+l}=U_{1}$ and the knot sequence $\left\{v_{j}\right\}_{1=1}^{q+l}$ satisfying $L_{2}=v_{1}=\cdots=v_{l}<v_{l+1}<\cdots<v_{q}<v_{q+1}=\cdots=v_{q+l}=U_{2}$, respectively. Then if $C 6$ in the main paper holds, $\log N_{[]}\left(\epsilon, \Theta_{\delta},\|\cdot\|_{\infty}\right) \leq K p q \log (\delta / \epsilon)$, for some constant $K>0$ and $\epsilon<\delta$.

Proof. First, we show that for any $\phi \in \Theta_{\delta}$, it is true that

$$
\sum_{i=1}^{p} \sum_{j=1}^{q} \alpha_{i, j}^{2} \leq p q(K \delta)^{2}
$$

Denote $\langle f, g\rangle=\int f g$. For each $(i, j)$, by the properties of $B$-spline basis functions and $\mathrm{C} 6$ in main paper, some tedious algebra leads to a conclusion that there exists $\psi_{i, j}=\sum_{i^{\prime}=1}^{p} \sum_{j^{\prime}=1}^{q} \gamma_{i^{\prime} j^{\prime}} N_{i^{\prime}}^{(1), l} N_{j^{\prime}}^{(2), l}$, such that $\left\langle N_{i}^{(1), l} N_{j}^{(2), l}, \psi_{i, j}\right\rangle$ $=1$ and $\left\langle N_{i^{\prime}}^{(1), l} N_{j^{\prime}}^{(2), l}, \psi_{i, j}\right\rangle=0$ for $\left(i^{\prime}, j^{\prime}\right) \neq(i, j)$, and $\left\langle\psi_{i, j}, \psi_{i, j}\right\rangle<K_{l}$ with the constant $K_{l}$ depending on $l$. Hence for any $\phi \in \Theta_{\delta}$,

$$
\alpha_{i, j}^{2}=\left\langle\phi, \psi_{i, j}\right\rangle^{2} \leq\langle\phi, \phi\rangle\left\langle\psi_{i, j}, \psi_{i, j}\right\rangle \leq(K \delta)^{2},
$$

which immediately results in (0.12).

Let

$$
S=\left\{\underline{\alpha}=\left(\alpha_{1,1}, \cdots, \alpha_{p q}\right): \quad \sum_{i=1}^{p} \sum_{j=1}^{q} \alpha_{i j}^{2} \leq p q(K \delta)^{2}\right\} .
$$

Lemma 0.4 indicates that there exist $\epsilon$-balls $B_{1}, B_{2}, \cdots, B_{\left[\left(\frac{\delta}{\epsilon}\right)^{K p q}\right]}$ centered at $\underline{\alpha}^{(1)}=\left(\alpha_{1,1}^{(1)}, \cdots, \alpha_{p, q}^{(1)}\right), \underline{\alpha}^{(2)}=\left(\alpha_{1,1}^{(2)}, \cdots, \alpha_{p, q}^{(2)}\right), \cdots, \underline{\alpha}^{\left(\left[\left(\frac{\delta}{\epsilon}\right)^{K p q}\right]\right)}=$ $\left(\alpha_{1,1}^{\left(\left[\left(\frac{\delta}{\epsilon}\right)^{K p q}\right]\right)}, \cdots, \alpha_{p, q}^{\left(\left[\left(\frac{\delta}{\epsilon}\right)^{K p q}\right]\right)}\right)$, respectively, which cover $S$.

Let

$$
\psi^{(k)}(s, t)=\sum_{i=1}^{p} \sum_{j=1}^{q} \alpha_{i, j}^{(k)} N_{i}^{(1), l}(s) N_{j}^{(2), l}(t)
$$

and

$$
\Psi_{1}^{(k)}=\left\{\psi:\left\|\psi-\psi^{(k)}\right\| \leq \epsilon \text { and } \psi \in \Psi\right\}
$$

for $k=1, \cdots,\left[\left(\frac{\delta}{\epsilon}\right)^{K p q}\right]$, where $\Psi=\left\{\psi: \psi(s, t)=\sum_{i=1}^{p} \sum_{j=1}^{q} \alpha_{i, j} N_{i}^{(1), l}(s) N_{j}^{(2), l}(t)\right\}$. Then $\left\{\Psi_{1}^{(k)}\right.$ : $\left.k=1, \cdots,\left[\left(\frac{\delta}{\epsilon}\right)^{K p q}\right]\right\}$ constitute a set of $\epsilon$-balls for $\Psi$.

In what follows, we show that $\left\{\Psi_{1}^{(k)}: k=1, \cdots,\left[\left(\frac{\delta}{\epsilon}\right)^{K p q}\right]\right\}$ cover $\Theta_{\delta}$. 
For any $\psi(s, t)=\sum_{i=1}^{p} \sum_{j=1}^{q} \alpha_{i, j} N_{i}^{(1), l}(s) N_{j}^{(2), l}(t) \in \Theta_{\delta}$, its coefficients $\underline{\alpha}=\left(\alpha_{1,1}, \cdots, \alpha_{p, q}\right) \in S$ by $(0.12)$. By the fact that $\epsilon$-balls $B_{1}, B_{2}, \cdots$, $B_{\left[\left(\frac{\delta}{\epsilon}\right)^{K p q}\right]}$ cover $S$, there exists $m$ with $1 \leq m \leq\left[\left(\frac{\delta}{\epsilon}\right)^{K p q}\right]$, such that

$$
\left\|\underline{\alpha}-\underline{\alpha}^{(m)}\right\|_{\infty}=\max _{\substack{i=1, \cdots, p \\ j=1, \cdots, q}}\left|\alpha_{i, j}-\alpha_{i, j}^{(m)}\right| \leq \epsilon
$$

Hence, for any $(s, t) \in\left[L_{1}, U_{1}\right] \times\left[L_{2}, U_{2}\right]$,

$$
\begin{aligned}
\left|\psi^{(m)}(s, t)-\psi(s, t)\right| & =\left|\sum_{i=1}^{p} \sum_{j=1}^{q}\left(\alpha_{i, j}^{(m)}-\alpha_{i, j}\right) N_{i}^{(1), l}(s) N_{j}^{(2), l}(t)\right| \\
& \leq \max _{\substack{i=1, \cdots, p \\
j=1, \cdots, q}}\left|\left(\alpha_{i, j}^{(m)}-\alpha_{i, j}\right)\right| \sum_{i=1}^{p} \sum_{j=1}^{q} N_{i}^{(1), l}(s) N_{j}^{(2), l}(t) \\
& =\max _{\substack{i=1, \cdots, p \\
j=1, \cdots, q}}\left|\alpha_{i, j}^{(m)}-\alpha_{i, j}\right| \leq \epsilon .
\end{aligned}
$$

And it follows that

$$
\left\|\psi^{(m)}-\psi\right\| \leq \epsilon .
$$

This implies that $\left\{\Psi_{1}^{(k)}: k=1, \cdots,\left[\left(\frac{\delta}{\epsilon}\right)^{K p q}\right]\right\}$ cover $\Theta_{\delta}$. Hence the $\epsilon$-covering number of $\Theta_{\delta}$ is bounded by $\left[\left(\frac{\delta}{\epsilon}\right)^{K p q}\right]$, or $\log N\left(\epsilon, \Theta_{\delta},\|\cdot\|_{\infty}\right) \leq K p q \log (\delta / \epsilon)$. It is obvious that

$$
N_{[]}\left(2 \epsilon, \Theta_{\delta},\|\cdot\|_{\infty}\right) \leq N\left(\epsilon, \Theta_{\delta},\|\cdot\|_{\infty}\right) .
$$

Therefore, it follows that

$$
\log N_{[]}\left(\epsilon, \Theta_{\delta},\|\cdot\|_{\infty}\right) \leq K p q \log (\delta / \epsilon) .
$$

Lemma 0.6. $\Theta_{\delta}=\left\{\phi: \phi(s)=\sum_{i=1}^{p} \beta_{i} N_{i}^{l}(s),\|\phi\|_{\infty}<\delta\right\},\left\{N_{i}^{l}\right\}_{i=1}^{p}$ are the B-spline basis functions with the knot sequence $\left\{u_{i}\right\}_{i=1}^{p+l}$ satisfying $L=$ $u_{1}=\cdots=u_{l}<u_{l+1}<\cdots<u_{p}<u_{p+1}=\cdots=u_{p+l}=U$. Then under $C 6$ in the main paper, $\log N_{[]}\left(\epsilon, \Theta_{\delta},\|\cdot\|_{\infty}\right) \leq K p \log (\delta / \epsilon)$, for some constant $K>0$ and $\epsilon<\delta$.

Proof. The proof is exactly along the same lines of those for Lemma 0.5 , and thus is omitted. 
LEMMA 0.7. $\Lambda_{0}(s, t)$ and $\Lambda(s, t)$ are both partially nondecreasing functions in the domain $\left[L_{1}, U_{1}\right] \times\left[L_{2}, U_{2}\right]$ and they satisfy $\left\|\Lambda-\Lambda_{0}\right\|_{L_{2}(\mu)} \leq \eta$. If the following conditions (1) and (2) hold, then there exists a constant $K$ independent of $\Lambda$ such that

$$
\sup _{(s, t) \in\left[L_{1}, U_{1}\right] \times\left[L_{2}, U_{2}\right]}\left|\Lambda(s, t)-\Lambda_{0}(s, t)\right| \leq(\eta / K)^{1 / 2} .
$$

(1) $\Lambda_{0}(s, t)$ is differentiable in both $s$ and $t$ and there exists a constant $0<$ $f_{0}<\infty$ such that $1 / f_{0} \leq \partial \Lambda_{0}(s, t) / \partial s \leq f_{0}$ and $1 / f_{0} \leq \partial \Lambda_{0}(s, t) / \partial t \leq$ $f_{0}$ for any $(s, t) \in\left[L_{1}, U_{1}\right] \times\left[L_{2}, U_{2}\right]$.

(2) The probability measure $\mu$ associated with $L_{2}$-norm has mixed derivative $\frac{\partial^{2} \mu(s, t)}{\partial s \partial t}$ satisfying $\frac{\partial^{2} \mu(s, t)}{\partial s \partial t} \geq c_{0}$ for some positive $c_{0}$.

Proof. Suppose that $\left(s^{\prime}, t^{\prime}\right) \in\left[L_{1}, U_{1}\right] \times\left[L_{2}, U_{2}\right]$ satisfies

$$
\left|\Lambda\left(s^{\prime}, t^{\prime}\right)-\Lambda_{0}\left(s^{\prime}, t^{\prime}\right)\right| \geq(1 / 2) \sup _{(s, t) \in\left[L_{1}, U_{1}\right] \times\left[L_{2}, U_{2}\right]}\left|\Lambda(s, t)-\Lambda_{0}(s, t)\right| \equiv \xi / 2 .
$$

Then either $\Lambda\left(s^{\prime}, t^{\prime}\right) \geq \Lambda_{0}\left(s^{\prime}, t^{\prime}\right)+\xi / 2$ or $\Lambda_{0}\left(s^{\prime}, t^{\prime}\right) \geq \Lambda\left(s^{\prime}, t^{\prime}\right)+\xi / 2$. In the following, we only show the inequality for the first case, $\Lambda\left(s^{\prime}, t^{\prime}\right) \geq \Lambda_{0}\left(s^{\prime}, t^{\prime}\right)+$ $\xi / 2$, as the arguments are parallel for the second case.

There exists $h$ satisfying $\left(s^{\prime}+h, t^{\prime}+h\right) \equiv\left(s^{\prime \prime}, t^{\prime \prime}\right)$, such that $\Lambda_{0}\left(s^{\prime \prime}, t^{\prime \prime}\right)=$ $\Lambda_{0}\left(s^{\prime}, t^{\prime}\right)+\xi / 2$. Then 


$$
\begin{aligned}
\eta^{2} & \geq \int\left\{\Lambda(s, t)-\Lambda_{0}(s, t)\right\}^{2} d \mu(s, t) \\
& =\iint_{(s, t) \in\left[L_{1}, U_{1}\right] \times\left[L_{2}, U_{2}\right]}\left\{\Lambda(s, t)-\Lambda_{0}(s, t)\right\}^{2} \frac{\partial^{2} \mu(s, t)}{\partial s \partial t} d s d t \\
& \geq \int_{t^{\prime}}^{t^{\prime \prime}} \int_{s^{\prime}}^{s^{\prime \prime}}\left\{\Lambda(s, t)-\Lambda_{0}(s, t)\right\}^{2} \frac{\partial^{2} \mu(s, t)}{\partial s \partial t} d s d t \\
& \geq \int_{t^{\prime}}^{t^{\prime \prime}} \int_{s^{\prime}}^{s^{\prime \prime}}\left\{\Lambda_{0}\left(s^{\prime \prime}, t^{\prime \prime}\right)-\Lambda_{0}(s, t)\right\}^{2} \frac{\partial^{2} \mu(s, t)}{\partial s \partial t} d s d t \\
& \geq c_{0} \int_{t^{\prime}}^{t^{\prime \prime}} \int_{s^{\prime}}^{s^{\prime \prime}}\left\{\Lambda_{0}\left(s^{\prime \prime}, t^{\prime \prime}\right)-\Lambda_{0}(s, t)\right\}^{2} d s d t \\
& \left.=c_{0} \int_{t^{\prime}}^{t^{\prime \prime}} \int_{\Lambda_{0}\left(s^{\prime}, t\right)}^{\Lambda_{0}\left(s^{\prime \prime}, t\right)}\left\{\Lambda_{0}\left(s^{\prime \prime}, t^{\prime \prime}\right)-x\right)\right\}^{2} \frac{1}{\partial \Lambda_{0}(s, t) /\left.\partial s\right|_{s=f_{t}^{-1}(x)}} d x d t \\
& \left.\geq\left(c_{0} / f_{0}\right) \int_{t^{\prime}}^{t^{\prime \prime}} \int_{\Lambda_{0}\left(s^{\prime}, t\right)}^{\Lambda_{0}\left(s^{\prime \prime}, t\right)}\left\{\Lambda_{0}\left(s^{\prime \prime}, t^{\prime \prime}\right)-x\right)\right\}^{2} d x d t \\
& =\left(c_{0} / f_{0}\right) \int_{t^{\prime}}^{t^{\prime \prime}}\left\{\left(\Lambda_{0}\left(s^{\prime \prime}, t^{\prime \prime}\right)-\Lambda_{0}\left(s^{\prime}, t\right)\right)^{3} / 3-\left(\Lambda_{0}\left(s^{\prime \prime}, t^{\prime \prime}\right)-\Lambda_{0}\left(s^{\prime \prime}, t\right)\right)^{3} / 3\right\} d t,
\end{aligned}
$$

where $x=f_{t}(s) \equiv \Lambda_{0}(s, t)$. Therefore, by $a^{3}-b^{3}=(a-b)\left(a^{2}+a b+b^{2}\right) \geq$ $(a-b)\left(a^{2}+b^{2}\right)$ for $a b(a-b) \geq 0$, it follows that

$$
\begin{aligned}
\eta^{2} \geq \frac{c_{0}}{3 f_{0}} \int_{t^{\prime}}^{t^{\prime \prime}}\left(\Lambda_{0}\left(s^{\prime \prime}, t\right)-\Lambda_{0}\left(s^{\prime}, t\right)\right)[ & \left(\Lambda_{0}\left(s^{\prime \prime}, t^{\prime \prime}\right)-\Lambda_{0}\left(s^{\prime}, t\right)\right)^{2} \\
& \left.+\left(\Lambda_{0}\left(s^{\prime \prime}, t^{\prime \prime}\right)-\Lambda_{0}\left(s^{\prime \prime}, t\right)\right)^{2}\right] d t .
\end{aligned}
$$

Using Taylor expansion, there exists $w \in\left(s^{\prime}, s^{\prime \prime}\right)$, such that

$$
\Lambda_{0}\left(s^{\prime \prime}, t\right)-\Lambda_{0}\left(s^{\prime}, t\right)=\left(\partial \Lambda_{0}(w, t) / \partial s\right) h \geq h / f_{0} .
$$

Using Taylor expansion along $s$ and $t$, respectively, we have

$$
\begin{aligned}
\xi / 2 & =\Lambda_{0}\left(s^{\prime \prime}, t^{\prime \prime}\right)-\Lambda_{0}\left(s^{\prime}, t^{\prime}\right) \\
& =\Lambda_{0}\left(s^{\prime \prime}, t^{\prime \prime}\right)-\Lambda_{0}\left(s^{\prime \prime}, t^{\prime}\right)+\Lambda_{0}\left(s^{\prime \prime}, t^{\prime}\right)-\Lambda_{0}\left(s^{\prime}, t^{\prime}\right) \\
& \leq 2 h f_{0} .
\end{aligned}
$$

Combining (0.14) and (0.15) yields,

$$
\Lambda_{0}\left(s^{\prime \prime}, t\right)-\Lambda_{0}\left(s^{\prime}, t\right) \geq \frac{\xi}{4 f_{0}^{2}} .
$$


Finally, substituting (0.16) into (0.13), we obtain

$$
\begin{aligned}
\eta^{2} & \geq \frac{c_{0} \xi}{12 f_{0}^{3}} \int_{t^{\prime}}^{t^{\prime \prime}}\left[\left(\Lambda_{0}\left(s^{\prime \prime}, t^{\prime \prime}\right)-\Lambda_{0}\left(s^{\prime}, t\right)\right)^{2}+\left(\Lambda_{0}\left(s^{\prime \prime}, t^{\prime \prime}\right)-\Lambda_{0}\left(s^{\prime \prime}, t\right)\right)^{2}\right] d t \\
& \geq \frac{c_{0} \xi}{12 f_{0}^{3}} \int_{t^{\prime}}^{t^{\prime \prime}}\left(\Lambda_{0}\left(s^{\prime \prime}, t^{\prime \prime}\right)-\Lambda_{0}\left(s^{\prime \prime}, t\right)\right)^{2} d t \\
& =\frac{c_{0} \xi}{12 f_{0}^{3}} \int_{\Lambda_{0}\left(s^{\prime \prime}, t^{\prime}\right)}^{\Lambda_{0}\left(s^{\prime \prime}, t^{\prime \prime}\right)}\left(\Lambda_{0}\left(s^{\prime \prime}, t^{\prime \prime}\right)-x\right)^{2} \frac{1}{\partial \Lambda_{0}\left(s^{\prime \prime}, t\right) /\left.\partial t\right|_{t=g_{s^{\prime \prime}}^{-1}(x)}} d x \\
& \geq \frac{c_{0} \xi}{12 f_{0}^{4}} \int_{\Lambda_{0}\left(s^{\prime \prime}, t^{\prime}\right)}^{\Lambda_{0}\left(s^{\prime \prime}, t^{\prime \prime}\right)}\left(\Lambda_{0}\left(s^{\prime \prime}, t^{\prime \prime}\right)-x\right)^{2} d x=\frac{c_{0} \xi}{12 f_{0}^{4}}\left(\Lambda_{0}\left(s^{\prime \prime}, t^{\prime \prime}\right)-\Lambda_{0}\left(s^{\prime \prime}, t^{\prime}\right)\right)^{3} / 3 \\
& \geq \frac{c_{0} \xi^{4}}{2304 f_{0}^{10}},
\end{aligned}
$$

where $x=g_{s^{\prime \prime}}(t) \equiv \Lambda_{0}\left(s^{\prime \prime}, t\right)$. This yields the stated conclusion with $K \equiv$ $\sqrt{c_{0} /\left(2304 f_{0}^{10}\right)}$.

\section{REFERENCES}

[1] De Boor, C. (2001). A Practical Guide to Splines. Springer, New York.

[2] Shen, X. and Wong, W. H. (1994). Convergence Rate of Sieve Estimates. Ann. Statist. 22 580-615. 